Eva Best/Martin Weth

Geschäftsprozesse optimieren 
Eva Best/Martin Weth

\section{Geschäftsprozesse optimieren}

Der Praxisleitfaden

für erfolgreiche Reorganisation 
Bibliografische Information Der Deutschen Bibliothek

Die Deutsche Bibliothek verzeichnet diese Publikation in der Deutschen Nationalbibliografie; detaillierte bibliografische Daten sind im Internet über <http://dnb.ddb.de> abrufbar.

1. Auflage Juni 2003

Alle Rechte vorbehalten

(c) Betriebswirtschaftlicher Verlag Dr. Th. Gabler/GWV Fachverlage GmbH, Wiesbaden 2003

Softcover reprint of the hardcover 1st edition 2003

Lektorat: Jens Kreibaum

Der Gabler Verlag ist ein Unternehmen der Fachverlagsgruppe BertelsmannSpringer.

www.gabler.de

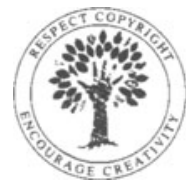

Das Werk einschließlich aller seiner Teile ist urheberrechtlich geschützt. Jede Verwertung außerhalb der engen Grenzen des Urheberrechtsgesetzes ist ohne Zustimmung des Verlags unzulässig und strafbar. Das gilt insbesondere für Vervielfältigungen, Übersetzungen, Mikroverfilmungen und die Einspeicherung und Verarbeitung in elektronischen Systemen.

Die Wiedergabe von Gebrauchsnamen, Handelsnamen, Warenbezeichnungen usw. in diesem Werk berechtigt auch ohne besondere Kennzeichnung nicht zu der Annahme, dass solche Namen im Sinne der Warenzeichen- und Markenschutz-Gesetzgebung als frei zu betrachten wären und daher von jedermann benutzt werden dürften.

Umschlaggestaltung: Nina Faber de.sign, Wiesbaden

Gedruckt auf säurefreiem und chlorfrei gebleichtem Papier

ISBN 978-3-322-94582-2

ISBN 978-3-322-94581-5 (eBook)

DOI 10.1007/978-3-322-94581-5 


\section{Vorwort}

Einer Untersuchung bei mittelständischen Unternehmen zufolge werden 36 Prozent der Arbeitszeit unproduktiv genutzt, weil die Prozesse unzureichend geplant und gesteuert werden. Demnach werden 78 Arbeitstage pro Mitarbeiter und Jahr verschwendet. Was für die ansonsten eher effizient geführten Mittelständler gilt, trifft unserer Erfahrung nach erst recht auf Großunternehmen zu. Kein Wunder, dass die Optimierung von Geschäftsprozessen nach wie vor eines der wichtigsten Managementthemen ist. Dies erfahren wir seit Jahren in unserem Berateralltag - und auch, dass bei Reorganisationen trotz exzellenter Management-Expertise oft Ratlosigkeit herrscht.

Die Capitum AG berät multinationale Unternehmen in Restrukturierungsvorhaben mit dem Ziel der Prozess- und Kostenoptimierung, in IT-Projekten zur Automatisierung von Prozessen und bei den daraus folgenden Change Management- und Personalmaßnahmen. Dabei stehen praxisnahe Konzepte wie die Begleitung des Kunden bei der Umsetzung und die Befähigung der Mitarbeiter, selbständig solche Projekte durchführen zu können, im Vordergrund. Wir möchten mit diesem Buch unsere Erfahrungen aus der Praxis an Manager weitergeben, die vor der Herausforderung einer umfassenden Reorganisation stehen. Die hier beschriebene Vorgehensweise haben wir kontinuierlich in unseren Beratungsprojekten erprobt und seit Jahren in Management-Trainings unseren Klienten vermittelt.

Insgesamt haben vier Partner der Capitum AG ihr Praxis-Know-how eingebracht: Ralf Behnstedt, Eva Best, Günther Eufinger und Martin Weth. Alle verfügen sowohl als Mitarbeiter wie auch als Berater von Großunternehmen über mehrjährige Erfahrung in Reorganisationsprojekten.

Unser herzlicher Dank gilt allen, die uns zahlreiche Anregungen zu diesem Buch gegeben, unermüdlich die anstrengende Arbeit des Korrekturlesens übernommen und uns nicht zuletzt den Rücken freigehalten haben.

Frankfurt am Main im Juni 2003

Ralf Behnstedt, Eva Best, Günther Eufinger, Martin Weth 


\section{Inhaltsverzeichnis}

Vorwort .5

1 Vorbereitung - Ein guter Anfang ist die Hälfte des Ganzen.............................. 11

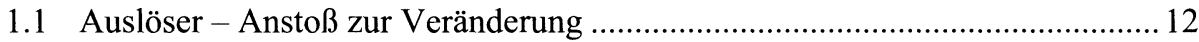

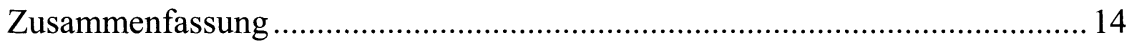

1.2 Vision - Der Reorganisation ein Leitbild geben .......................................... 15

1.2.1 Den Namen der Reorganisation zum Programm erklären..................... 15

1.2.2 Der Weg zur richtigen Vision ........................................................ 16

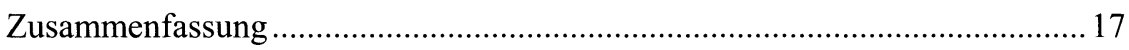

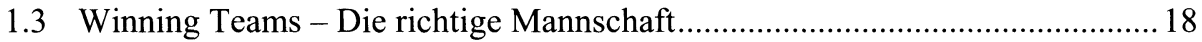

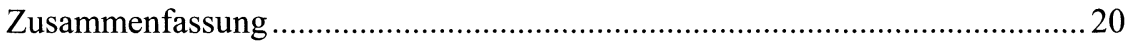

1.4 Vorgehensweise - Mit System zum Erfolg ............................................ 21

2 Potenzialanalyse - Wer den eigenen Ausgangspunkt nicht kennt, dem nutzt die

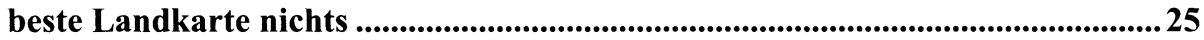

2.1 Analyse der Kunden und Wettbewerber - Blick nach außen .......................... 26

2.1.1 Wer sind unsere Wettbewerber? ............................................................26

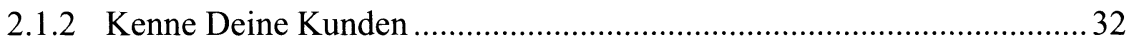

Zusammenfassung .......................................................................... 48

2.2 Analyse der eigenen Kernkompetenzen - Wo sind wir gut? ..........................50

2.2.1 Generierung von Mehrwert für den Kunden ...................................... 50

2.2.2 Ansatzpunkte für die Prozessoptimierung.......................................... 53

2.2.3 Potenzielle Outsourcing-Kandidaten...............................................5

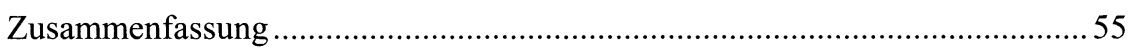

2.3 Prozessanalyse - Nur wer in Prozessen denkt, blickt durch .............................56

2.3.1 Vorbereitung der Prozessanalyse ......................................................5 57

2.3.2 Durchführung der Prozessanalyse ...................................................... 62

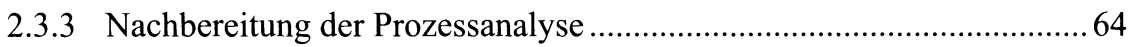

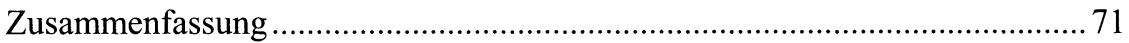

2.4 Problemdiagnose - Wo die größten Brocken liegen ...................................... 72

2.4.1 Identifikation von Schwachstellen ................................................... 72

2.4.2 Aufdecken des Ursachen-Wirkungs-Zusammenhangs........................80 
2.4.3 Identifikation des wirkungsvollsten Stellhebels für die Prozessoptimierung...................................................................... 83

2.4.4 Erste Erfolge durch Sofortmaßnamen ............................................. 87

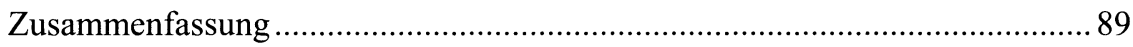

2.5 Zielformulierung - Was nicht gemessen wird, wird nicht getan .....................89

2.5.1 Typische Schwachstellen bei der Zielformulierung ............................. 90

2.5.2 Was wohlformulierte Ziele ausmacht.............................................. 92

2.5.3 Wie können Sie die Plausibilität der Ziele überprüfen? ....................... 97

2.5.4 Der Weg zu wohlformulierten Zielen ............................................ 101

3 Redesign - Gratwanderung zwischen Kreativität und Faustregeln ................... 103

3.1 Brainstorming - Ideenfelder für kreative Ansätze......................................... 104

3.2 Optimierung - Entwicklung von Redesign-Maßnahmen ............................ 107

3.2.1 Eliminieren überflüssiger Prozessschritte ....................................... 107

3.2.2 Parallelisieren von Prozessschritten .................................................. 110

3.2.3 Sicherstellen der Prozessqualität ..................................................... 112

3.2.4 Standardisieren von Prozessen .......................................................... 116

3.2.5 Automatisieren von Prozessen ......................................................... 120

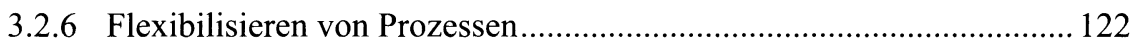

3.2.7 Optimieren der Schnittstellen zu Kunden ........................................ 127

3.2.8 Optimieren der Schnittstellen zu Lieferanten.................................... 132

3.2.9 Entwurf der Organisationsstruktur ..................................................... 134

3.2.10 Outsourcing von Prozessen .............................................................. 138

3.3 Target Activity Grid - Ein Instrument, um das Redesign wirksam zu

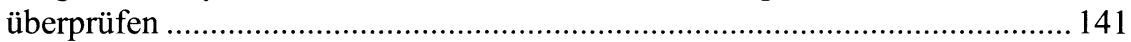

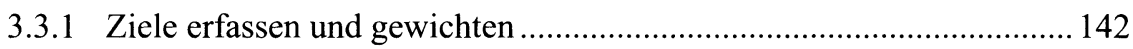

3.3.2 Maßnahmen definieren und erfassen................................................. 144

3.3.3 Maßnahmen im Target Activity Grid bewerten ................................ 146

3.3.4 Ziel-Index und Maßnahmen-Index ableiten ..................................... 146

3.3.5 Welchen Beitrag leistet das Target Activity Grid?............................ 151

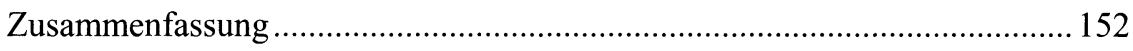

4 Umsetzung - Die neuen Prozesse in der Organisation zum Laufen bringen .... 155

4.1 Unternehmenskultur - Grenzen der Veränderung .................................... 156

Zusammenfassung ................................................................................... 158

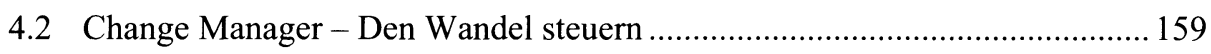




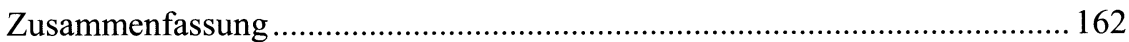

4.3 Kraftfeld - Im Netz der Beziehungen....................................................... 162

4.3.1 Beziehungsnetz - Stolpersteine erkennen ..................................... 164

4.3.2 Beziehungsmatrix - Einfluss messen ............................................ 166

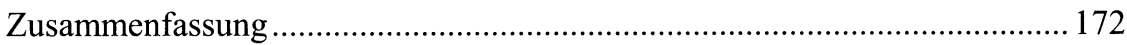

4.4 Umgang mit Widerstand - Gegenwind erzeugt Auftrieb ............................ 173

4.4.1 Keine Veränderung ohne Widerstand?.......................................... 173

4.4.2 Warum wir Widerstand leisten..................................................... 175

4.4.3 Widerstand erkannt - Gefahr gebannt? .......................................... 177

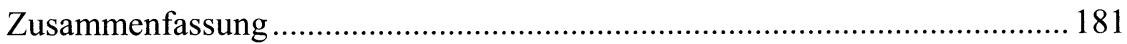

5 Nachbereitung - Erfolg messen und Wissen konservieren ................................ 183

5.1 Erfolgsmessung - Was hat das Ganze gebracht? ........................................ 183

Zusammenfassung .............................................................................. 184

5.2 Wissensmanagement - Erfahrungen aus dem Projekt weitergeben ................ 185

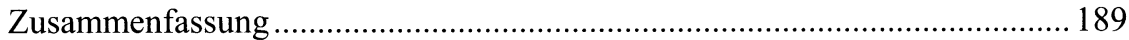

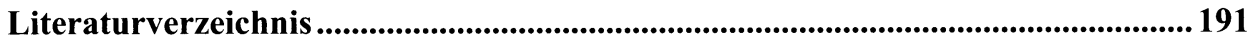

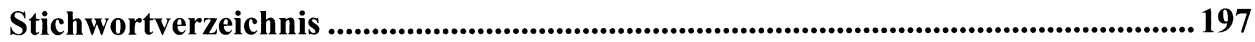

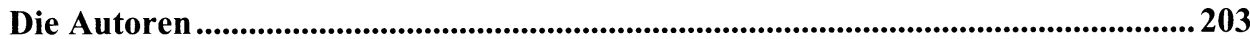

